\title{
How Influenza Vaccination Rate Variation Could Inform Pandemic-Era Vaccination Efforts
}

J Gen Intern Med 35(11):3401- 3

DOI: $10.1007 / \mathrm{s} 11606-020-06129-\mathrm{x}$

(C) Society of General Internal Medicine 2020

\section{INTRODUCTION}

With the coronavirus disease 2019 (COVID-19) pandemic persisting and an influenza season looming, preparing a public health strategy to approach herd immunity to influenza is critical to preserving health care capacity and saving lives. Influenza led to 45 million symptomatic cases and 61,000 deaths in the 2017-2018 season, but under half of US adults and two-thirds of children are vaccinated. ${ }^{1,2}$ The Centers for Disease Control and Prevention (CDC) recommends all persons 6 months of age or older without contraindications receive the influenza vaccine annually, especially those at higher risk for complications (e.g., older age, underlying medical conditions). ${ }^{2}$ Expanding upon earlier research on influenza vaccination disparities in $2011-2012,{ }^{3}$ we use 2018 data to provide updated vaccination rates and identify rate variations informative to public health efforts to control influenza transmission and develop an outreach strategy for COVID-19 and other pandemic-related vaccination.

\section{METHODS}

We analyzed rates of self-reported influenza vaccination in the last 12 months recorded in the 2018 wave of the Behavioral Risk Factor Surveillance System (BRFSS), an annual national survey of 400,000+ US adults conducted by the CDC and state health departments. The 2018 data capture vaccinations for late 2017-2018 and early 2018-2019 influenza seasons. We report vaccination rates nationally and by state, age, race, sex, annual household income, chronic condition, health insurance (yes/no), and personal doctor (yes/ no) subgroups weighted by BRFSS survey weights for national representativeness. Chronic conditions were defined from self-reports if respondents were ever told they had a heart attack, angina or coronary heart disease, asthma, any cancer other than skin cancer, chronic obstructive pulmonary disorder or emphysema or chronic bronchitis, arthritis, kidney disease, or diabetes. Multivariable logistic

Received June 22, 2020

Accepted August 10, 2020

Published online August 28, 2020 regression was used to test for independent associations between factors of interest and odds of having received vaccination.

\section{RESULTS}

Data on influenza vaccination were available for $95.3 \%$ of 437,436 BRFSS respondents. Nationally, an estimated 33.2\% of adults were vaccinated. Vaccination rates were higher among older, white and Asian, female, and higher income adults and those with chronic conditions (Table 1). In multivariable regression, these factors were all independently associated with higher odds of vaccination (Table 1). Ages $75+$ had three times higher adjusted odds of vaccination than ages 18-24. Black and Native American adults both had $17 \%$ lower odds of vaccination than white adults. Having health insurance and having a personal doctor were both independently associated with two times greater odds of being vaccinated. Comparing states, there was variation in weighted vaccination rates ranging from $26.4 \%$ in Texas to $44.2 \%$ in the District of Columbia (Fig. 1).

\section{DISCUSSION}

Only one-third of US adults received the influenza vaccination in 2018. Several demographic factors including male sex, Black and Native American races, and uninsured status predicted lower rates. In contrast, factors that elevate risk of complications, such as older age and underlying medical conditions, predicted higher rates. Still, no subgroup exceeded a $60 \%$ vaccination rate, far short of the $80 \%$ that is considered sufficient to reach herd immunity and the US government's Healthy People goal. ${ }^{4}$ States with lower vaccination rates might especially benefit from more intensive public health interventions.

The higher influenza vaccination rate in those with a personal doctor highlights the essential role primary care practices could play in facilitating vaccination. Prior research has identified patient outreach, clinician reminders, and financial incentives as promising clinical interventions to improve vaccination rates. ${ }^{5}$ Primary care visits could also provide opportunities to address commonly cited concerns about side effects, effectiveness, and vaccine-acquired infection in the $41 \%$ of Americans not intending to get vaccinated. ${ }^{6}$

Policy action is also worth consideration. Higher vaccination in insured adults suggests that reducing uninsurance 
Table 1 Rates of Influenza Vaccination Nationally and Stratified by Subgroups $(n=416,935)$

\begin{tabular}{|c|c|c|c|c|c|}
\hline Characteristic & No. vaccinated & Unweighted \% vaccinated & Weighted \% vaccinated & Adj. odds ratio (SE) ${ }^{c}$ & $p$ value \\
\hline National & 164092 & 39.4 & 33.2 & - & - \\
\hline \multicolumn{6}{|l|}{ Age groups ${ }^{\mathrm{a}}$} \\
\hline $18-24$ & 5537 & 22.6 & 21.6 & Ref & \\
\hline $25-34$ & 10602 & 24.1 & 22.5 & $1.01(0.04)$ & 0.725 \\
\hline $35-44$ & 13,500 & 27.6 & 25.2 & $1.02(0.04)$ & 0.530 \\
\hline $45-54$ & 18,612 & 30.5 & 28.9 & $1.12(0.04)$ & 0.004 \\
\hline $55-64$ & 33,669 & 39.3 & 38.4 & $1.61(0.06)$ & $<0.001$ \\
\hline $65-74$ & 43,598 & 51.2 & 51.0 & $2.48(0.09)$ & $<0.001$ \\
\hline $75+$ & 35,974 & 59.3 & 58.3 & $3.38(0.14)$ & $<0.001$ \\
\hline \multicolumn{6}{|l|}{ Race/Ethnicity } \\
\hline White & 131632 & 41.5 & 36.1 & Ref & \\
\hline Black & 11494 & 33.9 & 27.8 & $0.83(0.02)$ & $<0.001$ \\
\hline Asian & 3815 & 38.3 & 38.3 & $1.54(0.08)$ & $<0.001$ \\
\hline American Indian/Alaskan Native & 2833 & 35.6 & 29.3 & $0.83(0.05)$ & 0.003 \\
\hline Hispanic & 10036 & 28.9 & 25.3 & $1.05(0.03)$ & 0.120 \\
\hline Other race & 4282 & 31.7 & 28.5 & $0.88(0.04)$ & 0.004 \\
\hline \multicolumn{6}{|l|}{$\operatorname{Sex}^{\mathrm{a}}$} \\
\hline Male & 68986 & 36.7 & 30.7 & Ref & \\
\hline Female & 94820 & 41.6 & 35.7 & $1.17(0.02)$ & $<0.001$ \\
\hline \multicolumn{6}{|l|}{ Household income $^{\mathrm{a}}$} \\
\hline$<\$ 15,000$ & 11351 & 33.9 & 29.6 & Ref & \\
\hline$<\$ 25,000$ & 19934 & 35.7 & 29.6 & $0.99(0.03)$ & 0.767 \\
\hline$<\$ 35,000$ & 13766 & 37.7 & 30.7 & $0.99(0.04)$ & 0.765 \\
\hline$<\$ 50,000$ & 18537 & 38.5 & 31.5 & $1.01(0.04)$ & 0.728 \\
\hline \multirow{2}{*}{\multicolumn{6}{|c|}{ Chronic conditions ${ }^{b}$}} \\
\hline & & & & & \\
\hline 0 & 58055 & 31.6 & 26.8 & Ref & \\
\hline 1 & 51193 & 41.9 & 36.3 & $1.16(0.02)$ & $<0.001$ \\
\hline 2 & 28967 & 48.0 & 43.1 & $1.30(0.03)$ & $<0.001$ \\
\hline 3 & 14336 & 50.7 & 47.2 & $1.42(0.05)$ & $<0.001$ \\
\hline $4+$ & 11255 & 52.7 & 52.1 & $1.71(0.07)$ & $<0.001$ \\
\hline \multicolumn{6}{|l|}{ Insurance status $^{\mathrm{a}}$} \\
\hline Uninsured & 5224 & 16.1 & 14.0 & Ref & \\
\hline Insured & 156809 & 41.6 & 36.0 & $1.97(0.07)$ & $<0.001$ \\
\hline \multicolumn{6}{|l|}{ Personal doctor } \\
\hline No & 13068 & 19.4 & 16.4 & Ref & \\
\hline Yes & 148495 & 43.6 & 38.3 & $1.91(0.05)$ & $<0.001$ \\
\hline
\end{tabular}

${ }^{a}$ Number of don't know, refused, or missing: age groups (7291), racelethnicity (0), sex (894), income (76118), insurance status (1958), personal doctor or health care provider (2746)

${ }^{b}$ Some observations had data for only some chronic conditions: missing all (23), missing at least one condition (9475). Missing data were treated as 0 (no) in the summation of chronic conditions calculation

${ }^{c}$ Multivariable logistic regression $(n=390,000)$ of whether a respondent had the influenza vaccination in the last 12 months $(0 / 1)$ adjusted for state, age group, racelethnicity, sex, household income category, number of chronic conditions, insurance status, and having at least one personal doctor or healthcare provider. Model excluded observations with unknown/refused/missing values for the included variables. Coefficients for states are not shown. SE, standard error

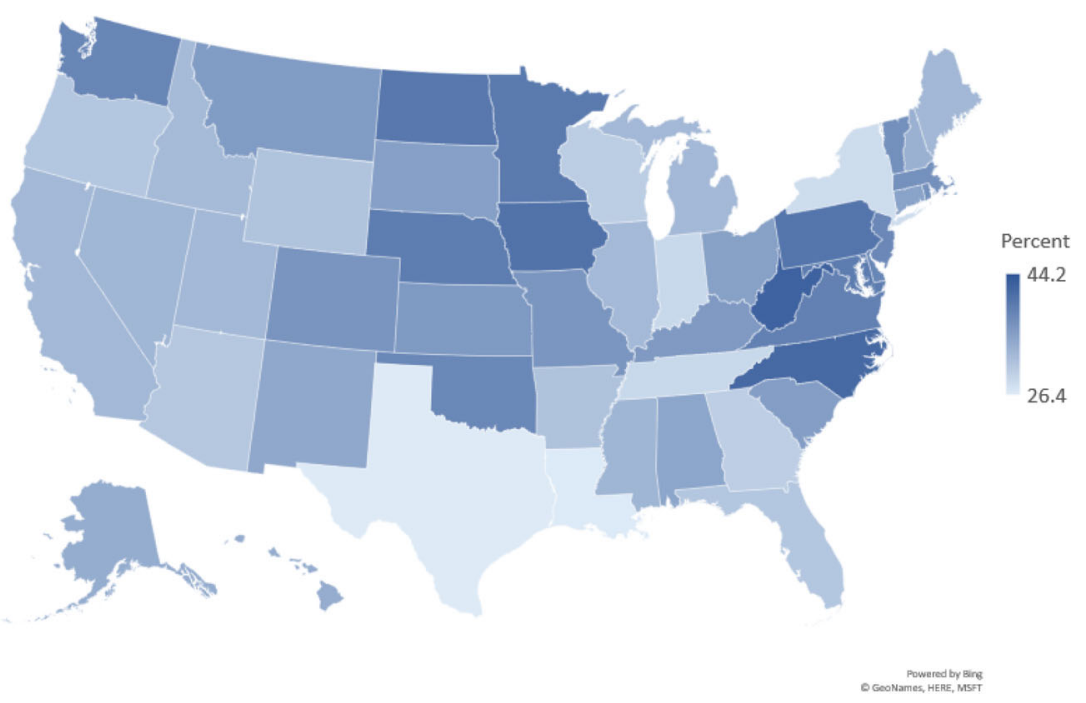

Fig. 1 Rates of influenza vaccination by US state weighted by BRFSS survey weights. The values for each state are available upon request. 
could, by increasing access to care, improve vaccination rates. Offering vaccinations at no out-of-pocket cost would eliminate the financial barrier for the uninsured especially. Pharmacies, including those inside supermarkets, could be incentivized to play larger roles in influenza vaccination. The concurrent COVID-19 pandemic also raises the issue of whether vaccination for influenza, COVID-19 (when a vaccine becomes available), or both should be mandated. While a federal mandate may be difficult politically, private organizations like colleges and employers could condition service utilization or employment on vaccination. Public health agencies might partner with community organizations in underserved communities to ensure culturally and linguistically appropriate outreach.

A persistently low influenza vaccination rate will have serious implications on health care capacity and mortality in the looming influenza season with a coinciding COVID-19 pandemic. These data inform where public health agencies and health care providers might focus resources towards reaching both higher and lower risk groups to bring the US closer to herd immunity.

Brandon W. Yan, $B A^{1}$

Frank A. Sloan, $P h D^{2}$

R. Adams Dudley, $M D M B A^{1,3}$

${ }^{1}$ Philip R. Lee Institute for Health Policy Studies and School of Medicine, University of California, San Francisco (UCSF),

San Francisco, CA, USA

${ }^{2}$ Department of Economics, Sanford School of Public Policy, and Margolis Center for Health Policy, Duke University,

Durham, NC, USA
${ }^{3}$ School of Medicine, School of Public Health, and Institute for Health Informatics, University of Minnesota,

Minneapolis, MN, USA

Corresponding Author: Brandon W. Yan, BA; Philip R. Lee Institute for Health Policy Studies and School of Medicine, University of California, San Francisco (UCSF)San Francisco, CA, USA (e-mail: Brandon.Yan@ucsf.edu).

Compliance with Ethical Standards: Conflict of Interest The authors have no conflicts of interest to report.

\section{REFERENCES}

1. Disease Burden of Influenza. Centers for Disease Control and Prevention. Published January 10, 2020. Accessed Mar 25, 2020. https://www.cdc. gov/flu/about/burden/index.html

2. Flu Vaccination Coverage, United States, 2018-19 Influenza Season. Centers for Disease Control and Prevention; 2019. Accessed Mar 17 2020. https://www.cdc.gov/flu/fluvaxview/coverage-1819estimates.htm

3. Lu D, Giao Y, Brown NE, Wang J. Racial and Ethnic Disparities in Influenza Vaccination among Adults with Chronic Medical Conditions Vary by Age in the United States. PLoS One 2017;12(1):e0169679. https://doi. org/10.1371/journal.pone.0169679

4. Plans-Rubió $\mathbf{P}$. The vaccination coverage required to establish herd immunity against influenza viruses. Prev Med 2012;55(1):72-77. https:// doi.org/10.1016/j.ypmed.2012.02.015

5. Lau D, Hu J, Majumdar SR, Storie DA, Rees SE, Johnson JA. Interventions to Improve Influenza and Pneumococcal Vaccination Rates Among Community-Dwelling Adults: A Systematic Review and MetaAnalysis. Ann Fam Med 2012;10(6):538-546. https://doi.org/10.1370/ afm. 1405

6. 41 Percent of Americans Do Not Intend to Get a Flu Shot this Season. NORC at the University of Chicago. Published December 5, 2018. Accessed Jun 22, 2020. https://www.norc.org/NewsEventsPublications/ PressReleases/Pages/41-percent-of-americans-do-not-intend-to-get-a-flushot.aspx

Publisher's Note: Springer Nature remains neutral with regard to jurisdictional claims in published maps and institutional affiliations. 